\title{
Domain Requirements and Sequence Specificity of DNA Binding for the Forkhead Transcription Factor FOXP3
}

\author{
Kian Peng Koh ${ }^{9}$, Mark S. Sundrud ${ }^{9}{ }^{\star}$, Anjana Rao* \\ Department of Pathology, Harvard Medical School and Immune Disease Institute, Boston, Massachusetts, United States of America
}

\begin{abstract}
The forkhead, winged-helix transcription factor FOXP3 is preferentially expressed in T regulatory (Treg) cells and is critical for their immunosuppressive function. Mutations that abolish FOXP3 function lead to systemic autoimmunity in mice and humans. However, the manner by which FOXP3 recognizes cognate DNA elements is unclear. Here we identify an in vitro optimized DNA sequence to assess FOXP3 DNA binding by electrophoretic mobility shift assay (EMSA). The optimized sequence contains two tandem copies of a core DNA element resembling, but not identical to, the canonical forkhead (FKH) binding element. The tandem nature of this optimized FOXP3-binding oligonucleotide suggests a requirement for multimerization, and EMSA experiments confirm that both the DNA-binding FKH domain and an intact leucine-zipper domain, which mediates homo-multimerization of FOXP3, are required for DNA binding. These results establish a practical framework for understanding the molecular basis by which FOXP3 regulates gene transcription and programs Treg suppressive function.
\end{abstract}

Citation: Koh KP, Sundrud MS, Rao A (2009) Domain Requirements and Sequence Specificity of DNA Binding for the Forkhead Transcription Factor FOXP3. PLoS ONE 4(12): e8109. doi:10.1371/journal.pone.0008109

Editor: Derya Unutmaz, New York University School of Medicine, United States of America

Received October 19, 2009; Accepted November 3, 2009; Published December 1, 2009

Copyright: @ 2009 Koh et al. This is an open-access article distributed under the terms of the Creative Commons Attribution License, which permits unrestricted use, distribution, and reproduction in any medium, provided the original author and source are credited.

Funding: This work was funded by NIH grants to A.R. (ROI AI48213 and R37 CA42471). M.S.S was supported by the Irvington Institute fellowship program of the Cancer Research Institute. The funders had no role in study design, data collection and analysis, decision to publish, or preparation of the manuscript.

Competing Interests: The authors have declared that no competing interests exist.

*E-mail: arao@idi.harvard.edu

a Current address: Section on Target Discovery, Tempero Pharmaceuticals, Cambridge, Massachusetts, United States of America

9 These authors contributed equally to this work.

\section{Introduction}

Forkhead box (FOX) transcription factors are a large and functionally diverse family of transcription factors, with over 100 members in mammals (reviewed in [1]). Named after the forkhead gene product in Drosophila melanogaster, the founding members of the mammalian FOX family belong to the hepatic nuclear factor-3 (HNF3/FOXA) family, which regulate the development of metabolic tissues such as the pancreas and liver [2,3]. Many FOX transcription factors are tissue-specific regulators of development (reviewed in [4]): hair formation and keratinocyte differentiation are regulated by Foxnl [5], cell growth and insulin responsiveness by Foxol [6], craniopharyngeal development by FOXE1 [4], speech and language patterning by FOXP2 [7], and auditory function by Foxil [8]. Additionally, several FOX proteins play key roles in the development, homeostasis and function of immune cells (reviewed in [9]). Foxo1, Foxo3 and Foxpl all regulate $\mathrm{B}$ cell ontogeny, possibly through direct transcriptional regulation of the Rag1/2 locus [10-13]. In T cells, genetic ablation of either Foxjl or Foxo3 precipitates a lymphoproliferative phenotype associated with variable autoimmune pathology $[14,15]$, suggesting that these FOX proteins negatively regulate $\mathrm{T}$ cell activation.

Foxp3 (denoted FOXP3 in humans) displays one of the more striking functions of a FOX protein within the immune system. FOXP3 is selectively expressed by a subset of CD4+ T cells, known as $\mathrm{T}$ regulatory (Treg) cells, which suppress effector $\mathrm{T}$ cell function in response to self or foreign antigens (reviewed in
$[9,16,17]$. FOXP3 is encoded on the X-chromosome, thus loss or mutation of FOXP3 is not deleterious in females. However, mutations within the FOXP3 gene in male infants are causally linked to IPEX (immune dysregulation, polyendocrinopathy, enteropathy, X-linked), a severe perinatal autoimmune syndrome resulting from defects in Treg development and consequent activation of conventional $\mathrm{T}$ cells with specificity for self-antigens $[18,19]$. IPEX patients develop lymphadenopathy, splenomegaly, hyper-IgE production, variable hyperglycemia and lymphocytic infiltrates into the lung, skin, pancreas and liver [18-20]. The autoimmune phenotype of IPEX patients is phenocopied in male scurfy (Foxp $3^{s f}$ ) mice, which harbor a spontaneous mutation in the Foxp3 gene [16,21,22]. Male mice in which Foxp3 is conditionally deleted in $\mathrm{T}$ cells using Cre recombinase expressed under the control of the CD4 promoter (CD4-Cre) develop a similar severe autoimmune phenotype [23], as do adult mice in which Foxp3expressing Treg cells are acutely ablated [24]. These observations have resulted in much focus on the transcriptional regulatory function of FOXP3.

FOXP3 contains a large $(\sim 181$ aa $)$ amino-terminal region required for transcriptional activation and repression, a central $\mathrm{C} 2 \mathrm{H} 2$ zinc-finger domain to which no specific function has yet been ascribed, a leucine-zipper domain implicated in multimer formation and suppressor function, and a C-terminal forkhead (FKH) domain that mediates DNA-binding by FOX proteins $[9,16]$. FOXP3 can associate with auxiliary transcription factors such as NFAT, AML1/Runx1, IRF4 (not shown to physically interact with Foxp3) and NF- $\kappa \mathrm{B}$ to drive the transcription of 
specific subsets of FOXP3 target genes [25-28]. These associations have been largely observed via co-immunoprecipitation and chromatin immunoprecipitation (ChIP) assays. ChIP assays can localize transcription factor binding to relatively large (200$500 \mathrm{bp}$ ) regions of DNA, and have proved useful in confirming or revealing target promoters likely to be directly regulated by FOXP3 and its transcriptional partners. Large-scale 'ChIP-chip' assays, in which DNA occupied by specific transcription factors is immunoprecipitated and hybridized to genome-wide tiling arrays, have been used to identify DNA elements likely to bind FOXP3 in vivo, either alone or in complex with transcriptional partners $[29,30]$. However, these analyses have yet to be confirmed by in vitro assays that directly assess FOXP3:DNA-binding.

Using a systematic series of electrophoretic mobility shift assays (EMSA), we have explored the basis for the sequence-specific DNA-binding by FOXP3. We show that a fragment lacking the first 181 amino acids of FOXP3 (Foxp3- $\Delta \mathrm{N}$ ) binds DNA far more robustly than full-length FOXP3. Efficient DNA binding by this fragment requires both the leucine zipper and FKH domains. The preferred oligonucleotide defined by EMSA assays as a highaffinity FOXP3-binding site contains two tandem FOXP elements, which are similar to, but somewhat divergent from, the classic forkhead-binding sites previously identified for HNF3/FOXA proteins. Based on the tandem nature of optimal FOXP3 binding sequences, together with the requirement for the leucine-zipper motif for DNA binding, we propose that FOXP3 binds DNA with high affinity as at least a dimer and that the N-terminal region has an autoinhibitory effect. Collectively, these results lay the foundation for understanding how FOXP3 controls the immunosuppressive transcriptional program of Treg cells.

\section{Methods}

\section{Plasmids}

cDNAs encoding full length mouse FoxplA (generous gift of Dr. Phil Tucker, University of Texas-Austin) or full length human FOXP3, as well as truncated and/or mutated versions were cloned into the expression vector pcDNA3.1(+). Point mutations/ deletions were generated in the constructs using the Quickchange site-directed mutagenesis system (Strategene).

\section{Electrophoretic Mobility Shift Assays}

The following oligonucleotide sequences were used as probes in gel-shift assays (one strand shown with putative binding sites underlined):

A: 5'-CAAGGTAAACAAGACAACACAAATA A-3'; A'(A1-A1): 5'-CAAGGTAAACAAGACA ACGTAAACAA-3'; A" (A2-A2): 5'-CAAGACA AATAAGACAACACAAATAA$3^{\prime}$

A'(AT): 5'-CAAGATAAACAAGACAACATAAACAA-3';

A'(GC): 5'-CAAG GCAAACAAGACAACGCAA ACAA-3';

$\mathrm{A}^{\prime}(\mathrm{AC}):$ 5'-CAAG $\overline{\mathrm{ACAAACA}} \mathrm{AGAC}$ AACACAAACAA$-3^{\prime}$;

A' (29 bp): 5'-CAAGGTA AAGAAGACAACGTAAACAAGTC-3';

A' (25 bp): 5'-CAAGGTAAAGAAGAGTAAACAAGTC-3';

A' (35 bp): 5'-CAAG $\overline{\text { GTAAACAAGACAACACG ATTGTA- }}$ AACAAGTC-3'

Single-stranded oligonucleotides containing the consensus Foxpl/ FOXP3 binding sites were annealed with their complementary strands and purified on $12 \%$ polyacrylamide gels for use as probes in electrophoretic mobility-shift assays (EMSA). Probes were endlabeled with $\gamma^{32}$ P-ATP using T4 polynucleotide kinase in accordance with manufacturers' instructions. In vitro-translated proteins were generated using the TNT reticulocyte lysate system (Promega).
Binding reactions were performed at room temperature for 20 minutes using $5 \mu \mathrm{l}$ of in vitro-translated proteins and approximately $10,000-20,000$ c.p.m. $(\sim 0.1-0.5 \mathrm{ng})$ of ${ }^{32} \mathrm{P}$-end labeled probes in $20 \mu$. The final concentration of components of the binding buffer for all EMSA experiments were: $12 \mathrm{mM}$ HEPES pH 7.5, $100 \mathrm{mM}$ $\mathrm{NaCl}, 1 \mathrm{mM}$ DTT, $1 \mathrm{mM}$ EDTA, $12 \%$ glycerol and $20 \mu \mathrm{g} / \mathrm{ml}$ poly $(\mathrm{dI})$-poly $(\mathrm{dC})$. DNA-protein complexes were separated from free probe by electrophoresis in a $5 \%$ polyacrylamide, TBE gel containing $1 \%$ glycerol. Dried gels were exposed to autoradiography film between 1 hour to overnight at room temperature. Quantification of band intensities were performed on autoradiograms from 1 hour exposures using the software ImageJ.

\section{Western Blot}

Equal quantities of in vitro-translated protein lysates were resolved by SDS-polyacrylamide gel electrophoresis and transferred to nitrocellulose membranes (Whatman). Immunoblots were performed using either a monoclonal antibody against HA (for HA-tagged Foxp1), a monoclonal antibody $1 \mathrm{G} 1$ raised against the FKH domain of Foxpl (generously provided by Dr. Philip Tucker) or a polyclonal rabbit antisera raised against full-length human FOXP3 (generously provided by Dr. Steven Ziegler). Antibodies were diluted in Tris-buffered saline containing $0.1 \%$ Tween-20 and 3\% non-fat dry milk. Secondary horseradish peroxidase-conjugated goat anti-mouse or anti-rabbit secondary antibodies (Sigma-Aldrich) were used to detect primary antibody binding, followed by detection with an enhanced chemiluminescence (ECL) reagent (Perkin-Elmer).

\section{Results}

All FOX transcription factors share a common winged-helix DNA-binding domain of approximately 100 amino acids known as the forkhead $(\mathrm{FKH})$ domain $[1,4,9]$. HNF3/FOXA proteins bind as monomers to DNA elements with the consensus sequence $5^{\prime}-$ ATAACT-3' [32,33]; however, primary sequence analyses of their FKH domains, and hence their putative sequence specificity for DNA, show a significant degree of divergence from the FKH domains of other FOX proteins [1]. Indeed FoxplA, a close relative of FOXP3, was found to prefer modified FKH/FOX DNA elements (5' TATTTg/aTg/aTT-3') or its complement, 5'AAc/tAc/tAAATA- 3 ') in a PCR-based site-selection assay from which the "A" oligonucleotide containing the preferred Foxpl binding site was derived [34].

We previously showed using a nonradioactive EMSA format - in which DNA and protein reactants are present at micromolar rather than nanomolar concentrations - that recombinant FOXP3-FKH domain expressed in bacteria bound very weakly on its own to the ARRE2 sequence from the mouse IL-2 promoter but formed a cooperative complex with recombinant NFAT1 DNA-binding domain on DNA [25]. Binding of the isolated FKH domain of FOXP3 to the ARRE2 sequence or the A oligonucleotide (see below) could not be detected in radioactive EMSA assays (data not shown), suggesting that other regions in FOXP3 are required for optimal DNA binding. To explore this possibility, we synthesized murine full-length HA-tagged FoxplA (referred to throughout as Foxp1), human full-length FOXP3, or defined fragments of FOXP3 (Figure 1A), by in vitro-transcription/translation in reticulocyte lysates. To compare the ability of these proteins to bind DNA in vitro, we used the A oligonucleotide (sequence shown in Figure 1B), which contains the Foxp 1 consensus sequence [34], as the starting probe in radioactive EMSA. All proteins were robustly expressed (Figure 1B, bottom). As expected, full-length Foxpl bound strongly to the A probe (Figure 1B, lane 2), but surprisingly, full-length FOXP3 did 
A

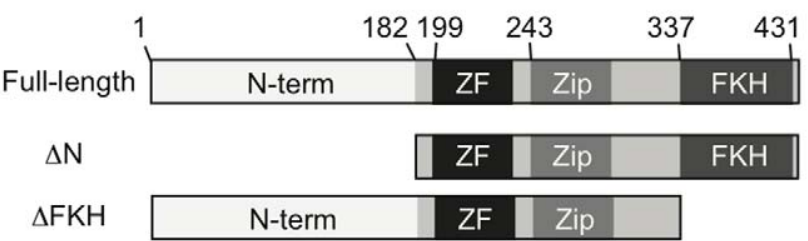

B

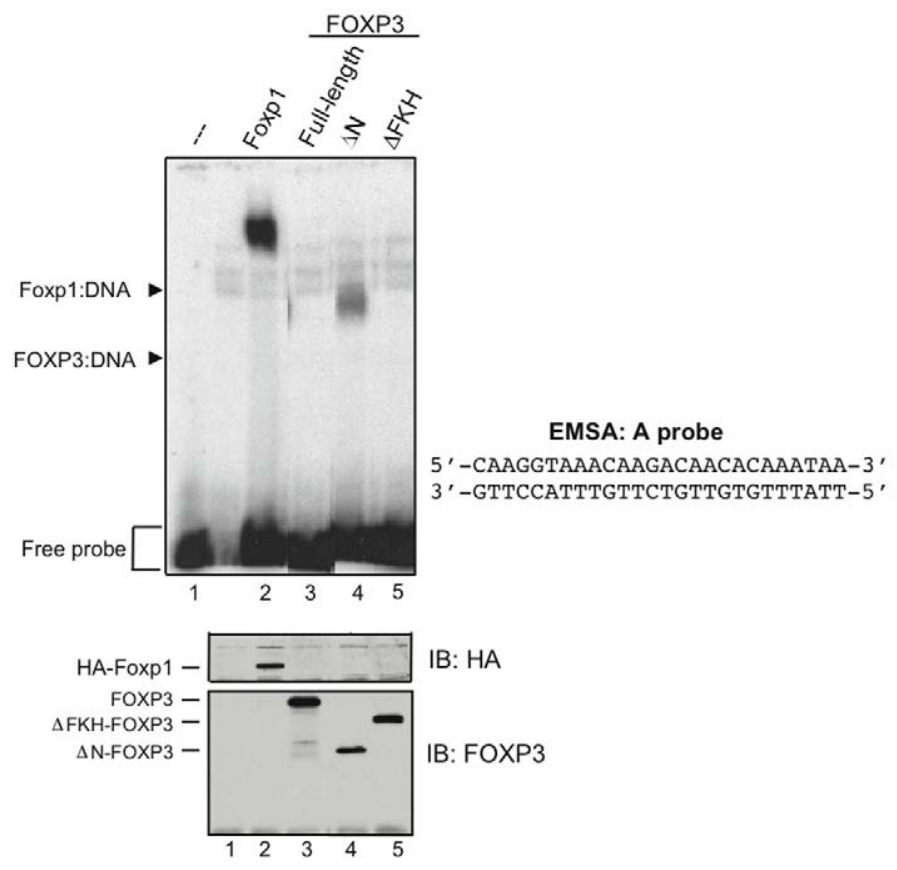

Figure 1. Deletion of the FOXP3 $\mathbf{N}$-terminal region allows binding to a Foxp1-like DNA element. (A) Fragments of human FOXP3 translated in vitro (see materials) for use in EMSA assays. Residue numbering is listed above full-length FOXP3. N-term - the N-terminal region (1-181) of FOXP3, ZF - zinc finger, Zip - leucine zipper, FKH - forkhead domain. (B) Top - Full-length Foxp1 or FOXP3 fragments were expressed and incubated with radiolabelled A probe. Arrowheads indicate Protein:DNA complexes and free probe. Bottom - Anti-HA monoclonal antibodies or FOXP3 antiserum were used to detect expression of Foxp1 or FOXP3 fragments, respectively, by western blotting. Arrowheads indicate the position of each construct. Data are representative of at least 2 independent experiments.

doi:10.1371/journal.pone.0008109.g001

not (lane 3). In contrast, a fragment lacking the first $181 \mathrm{~N}$-terminal amino acids of FOXP3, here designated FOXP3- $\Delta \mathrm{N}$ [25], bound to the A probe, although more weakly than that observed for Foxpl (Figure 1B, lane 4). Binding to the A probe was specific, as neither Foxpl nor FOXP3- $\Delta \mathrm{N}$ bound to a labeled oligonucleotide from the variable 1 region of the immunoglobulin promoter $(\mathrm{V} 1 \mathrm{P})$, which contains a canonical FOX consensus sequence defined for the HNF3/FOXA proteins (data not shown) [33]. FOXP3- $\Delta \mathrm{N}$ was thus used in subsequent experiments to define the DNA-binding specificity of FOXP3.

The weak binding of FOXP3- $\Delta \mathrm{N}$ to the A probe prompted us to derive an optimized sequence for FOXP3 DNA binding. Sequence inspection of the A probe revealed two potential FOXPbinding elements separated by a 7-nt spacer (Figure 2A). The $5^{\prime}$ element (5'-GTAAACA-3', here designated A1) matched a computationally-identified FOXP3 binding element obtained via ChIP-chip experiments (G/A T/c AAACA, Figure 2A) [30]. The 3' binding site (5'-AACACAAATA, here designated A2) was previously defined as the Foxpl consensus site (5'-AA C/t A C/t
AAATA, Figure 2A) [34]. FOXP3- $\Delta \mathrm{N}$ contains a leucine-zipper domain reported to mediate homotypic interactions, that is mutated in a subset of IPEX patients $[9,16]$, suggesting that the $\mathrm{A} 1$ and $\mathrm{A} 2$ sequences might interact independently with FOXP3 forkhead domains within a FOXP3 multimer. To test this hypothesis and determine whether FOXP proteins discriminated between these sites, we synthesized two new double-stranded oligonucleotides, $\mathrm{A}^{\prime}$ or $\mathrm{A}^{\prime \prime}$, containing two $\mathrm{A} 1$ or $\mathrm{A} 2$ elements, respectively (Figure 2A), and assessed their binding to in vitrotranslated Foxp1, Foxp1- $\Delta \mathrm{N}$, FOXP3, or FOXP3- $\Delta \mathrm{N}$ proteins in radioactive EMSA assays. For both Foxpl and FOXP3, the $\Delta \mathrm{N}$ versions bound DNA more effectively than the full-length proteins (Figure 2B), even though they were not over-expressed relative to the full-length proteins (Figure 2C). In fact, DNA binding by fulllength FOXP3 was not detectable with any of the three probes (Figure 2B). Both Foxp $1-\Delta \mathrm{N}$ and FOXP3- $\Delta \mathrm{N}$ displayed diminished binding to the $\mathrm{A}^{\prime \prime}$ (A2-A2) probe but enhanced binding to the $\mathrm{A}^{\prime}$ (Al-A1) probe, giving an order of preference for both proteins of $\mathrm{A}^{\prime}(\mathrm{A} 1-\mathrm{A} 1)>\mathrm{A}(\mathrm{A} 1-\mathrm{A} 2)>\mathrm{A}^{\prime \prime}(\mathrm{A} 2-\mathrm{A} 2)$ (Figure 2B). 
A
A1 site
A2 site
FOXP3 consensus Foxp1 consensus

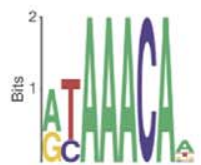
AACACAAATA
$t \quad t$
A (A1-A2) 5'-CAAGGTAAACAAGACAACACAAATAA-3'
$\mathrm{A}^{\prime}(\mathrm{A} 1-\mathrm{A} 1)$ 5' - CAAGGTAAACAAGACAACGTAAACAA-3'
A" (A2-A2) 5' - CAAGACAAATAAGACAACACAAATAA-3'

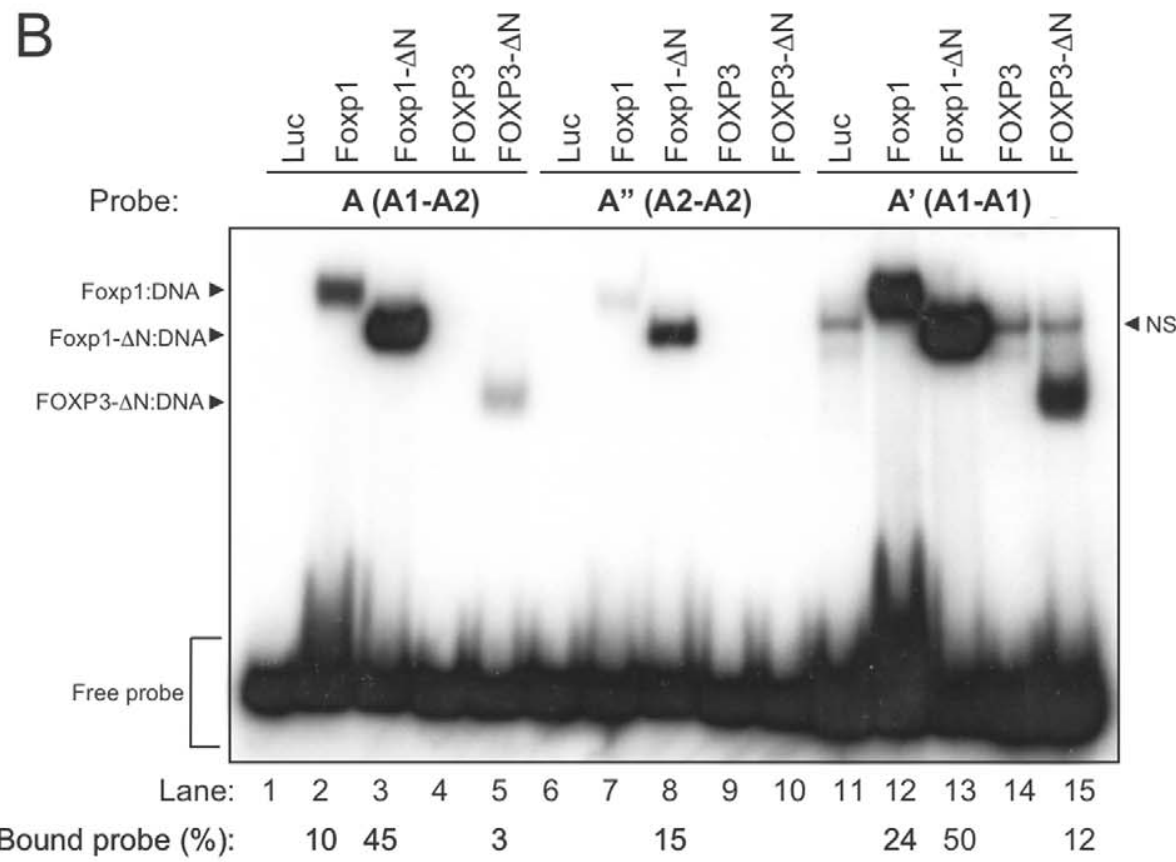

C

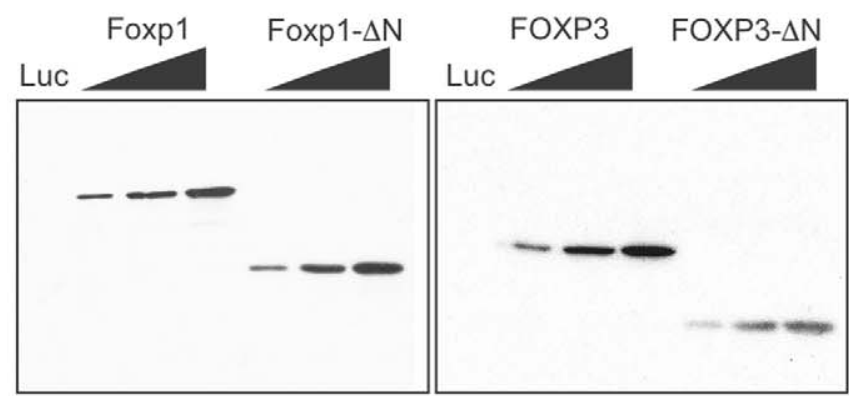

IB: Foxp1

IB: FOXP3

Figure 2. DNA binding specificities of Foxp1 and FOXP3. (A) Sequences of the A probe [34], $A^{\prime}$ and $A^{\prime \prime}$ oligonucleotides used for EMSA experiments. The $5^{\prime}$ putative FOXP3-binding site (A1) is similar to a predicted Foxp3 binding site [29,30] (see insert adapted from [30] below sequence text). The $3^{\prime}$ binding site (A2) represents the Foxp1 consensus site (blue text) as determined previously [34]. $A^{\prime}$ has two putative FOXP3binding sites (A1-A1). A" has two putative Foxp1 sites (A2-A2). (B) In vitro-translated firefly luciferase (Luc), Foxp1 (full-length or $\Delta N$ ) or FOXP3 (fulllength or $\Delta \mathrm{N}$ ) were incubated with each labeled probe as indicated. Protein:DNA complexes and free probe are indicated at left (by arrowhead in one case and a square bracket in the other). NS - non-specific. Quantification values of bound probe (shown as \% bound of total detected probe in each lane) were indicated below the lanes. (D) Expression of Foxp1 or FOXP3 constructs was evaluated by western blotting using anti-Foxp1 monoclonal or anti-FOXP3 polyclonal antisera respectively. Loading amounts of in vitro-translated lysates were $4 \mu \mathrm{l}$ for Luc and $1 \mu \mathrm{l}, 2 \mu \mathrm{l}$ and $4 \mu \mathrm{l}$ for the Foxp1 and FOXP3 constructs as in (C). Data are representative of at least two independent experiments.

doi:10.1371/journal.pone.0008109.g002 
The lack of detectable binding of FOXP3- $\Delta \mathrm{N}$ to the $\mathrm{A}^{\prime \prime}$ (A2-A2) probe, despite binding to $\mathrm{A}^{\prime}$ (A1-A1) and A (A1-A2), suggests that the Al sequence (GTAAACA) is essential for FOXP3 DNA binding. The diminished binding of full-length Foxpl and Foxpl- $\Delta \mathrm{N}$ to $\mathrm{A}^{\prime \prime}$ (A2-A2) was surprising, given that the probe contains the defined Foxp1 consensus element [34] duplicated in tandem. These results suggest that the strong binding of Foxpl to the A oligonucleotide was in fact facilitated by the presence of the Al element (GTAAACA) serving as a stronger Foxpl consensus site than A2 (ACAAATA).
Since the Al sequence (GTAAACA) is only one of four possible sequences derived from the computationally-identified FOXP3 consensus site (G/A T/c AAACA) [30], we repeated the EMSA assays using $\mathrm{A}^{\prime}$ (A1-A1) oligonucleotide probes that contained all the possible combinations of these preferred nucleotides: AT, AC, GT and GC (Figure $3 \mathrm{~A}$ ). FOXP3- $\Delta \mathrm{N}$ showed a strong preference for duplicated GTAAACA sequences, with binding affinity more than doubled compared to the original A probe containing only one copy of GTAAACA, or the oligonucleotide containing two ATAAACA

A

A (A1-A2) 5'-CAAGGTAAACAAGACAACACAAATAA-3' $A^{\prime}(\mathrm{GT}) \quad 5{ }^{\prime}$-CAAGGTAAACAAGACAACGTAAACAA-3' $\mathrm{A}^{\prime}(\mathrm{AT}) \quad 5^{\prime}$-CAAGATAAACAAGACAACATAAACAA-3' $A^{\prime}(\mathrm{GC}) \quad 5^{\prime}$-CAAGGCAAACAAGACAACGCAAACAA-3' $\mathrm{A}^{\prime}$ ( $\mathrm{AC}$ ) 5'-CAAGACAAACAAGACAACACAAACAA-3 '
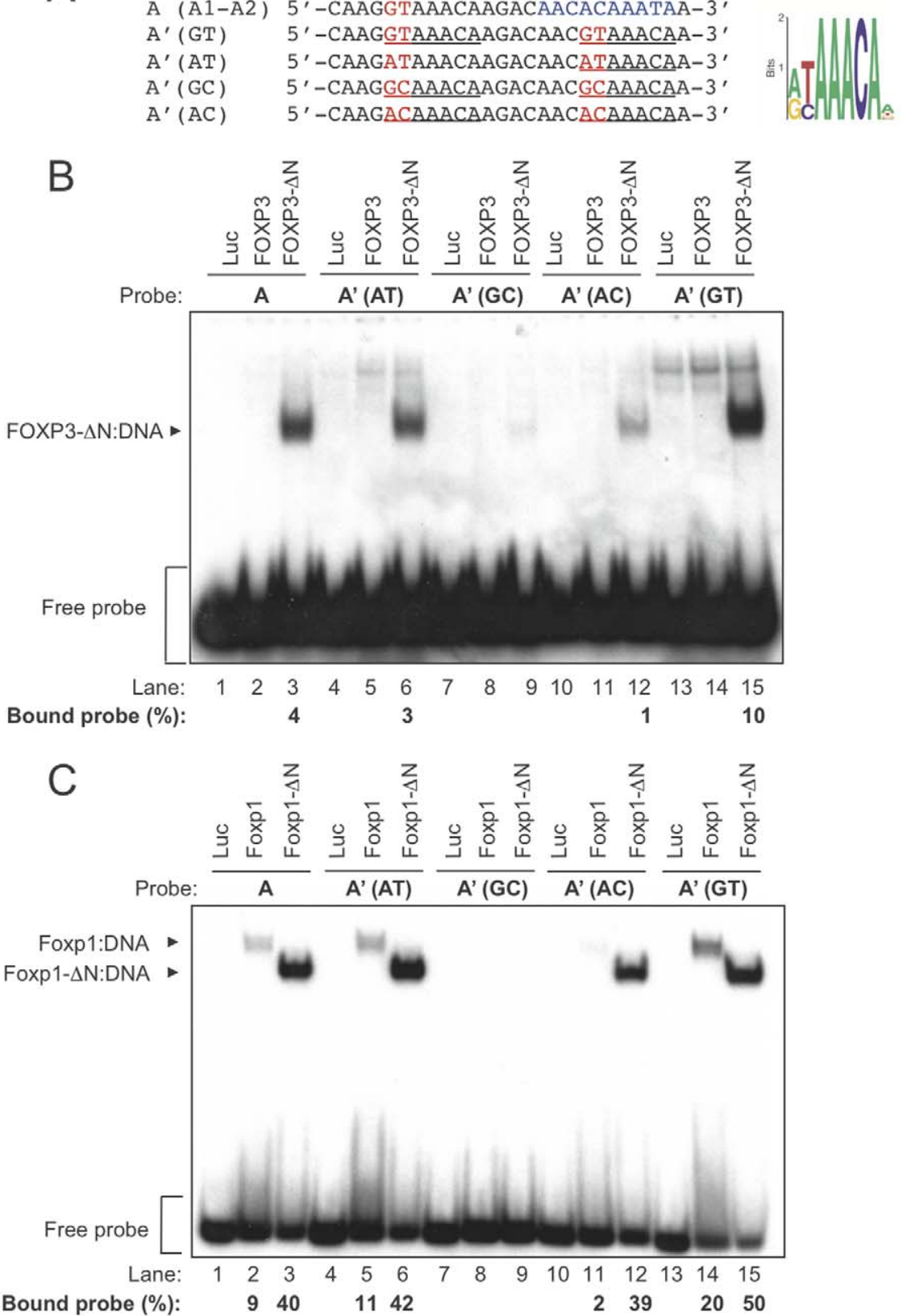

Figure 3. Definition of the FOXP3 consensus binding site. (A) Left - Using the $A^{\prime}$ oligonucleotide sequence as a reference, the two $5^{\prime}$ nucleotides were randomized within each of the two putative FOXP3 binding sites. Each FOXP3 binding site is underlined and the randomized $5^{\prime}$ dinucleotide motifs are indicated by red text. Right - Computationally-determined putative FOXP3 binding site as in the figure 2 legend. (B) In vitrotranslated firefly luciferase (Luc), full-length FOXP3 or FOXP3- $\Delta \mathrm{N}$ were incubated with each labeled probe as indicated. Protein:DNA complexes and free probe are indicated by arrowhead and square brackets as in the legend to Figure 2. (C) The same experiment as described above in Figure 3B was performed with in vitro-translated full-length Foxp1 or Foxp1- $\Delta \mathrm{N}$. Quantification values of bound probe were indicated below the lanes. These data represent at least 2 independent experiments.

doi:10.1371/journal.pone.0008109.g003 
sequences (Figure 3B). FOXP3- $\Delta \mathrm{N}$ binding to ACAAACA sequences was further decreased, and binding was altogether abolished to GCAAACA sequences (Figure 3B). These data suggest that FOXP3- $\Delta \mathrm{N}$ :DNA-binding is tightly regulated by the two $5^{\prime}$ nucleotides within its binding sites and gives an order of preference of GT $>$ AT $>$ AC (Figure 3B). Foxpl displayed similar preferences for its DNA-binding sites (Fig. 3C). In this case, however, the differential preference for GT, AT and AC was less pronounced, whereas binding to sequence starting with GC was again very weak. Collectively, these experiments define the core consensus binding element for both FOXP proteins as two tandem copies of the sequence 5'-GTAAACA-3'.

We next asked whether the spacing between the two core binding sites was important for FOXP3:DNA binding. For this we used synthetic oligonucleotides in which the $5^{\prime}$ ends of the two FOXP3 consensus elements were separated by 14 base-pairs (bp), as in the original A probe, or alternatively by 10 or $20 \mathrm{bp}$, corresponding to one or two complete turns of the DNA helix respectively, which would place the two sites on the same side of the DNA (Figure 4A). We found that shortening the spacer length between the two binding sites to 10 nucleotides, or lengthening the spacing to 20 nucleotides, increased FOXP3- $\Delta$ N DNA binding by $\sim 3$-fold (Figure $4 \mathrm{~B}$ ). In contrast, Foxpl- $\Delta \mathrm{N}$ preferred the longer spacing, with the 20-bp spacing somewhat preferred over the 14bp spacing originally selected by PCR-based site selection approaches (Figure 4B), but showed lower binding when the core sites were spaced by $10 \mathrm{bp}$. These data suggest that the optimal FOXP3 binding element contains two 5'-GTAAACA-3' sites

\section{A}

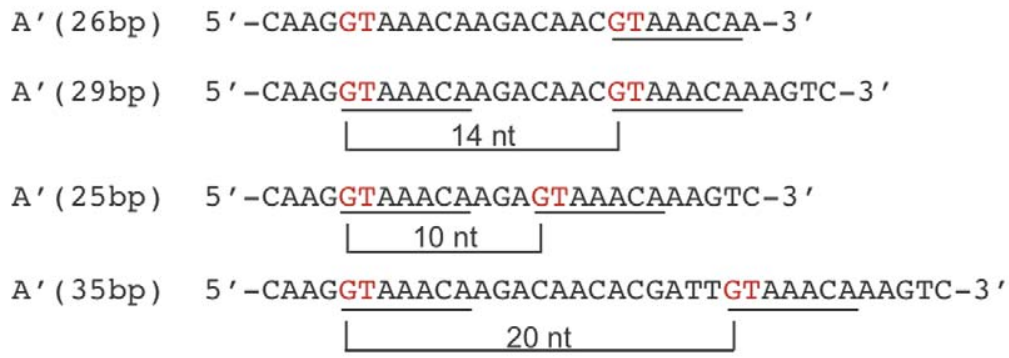

B

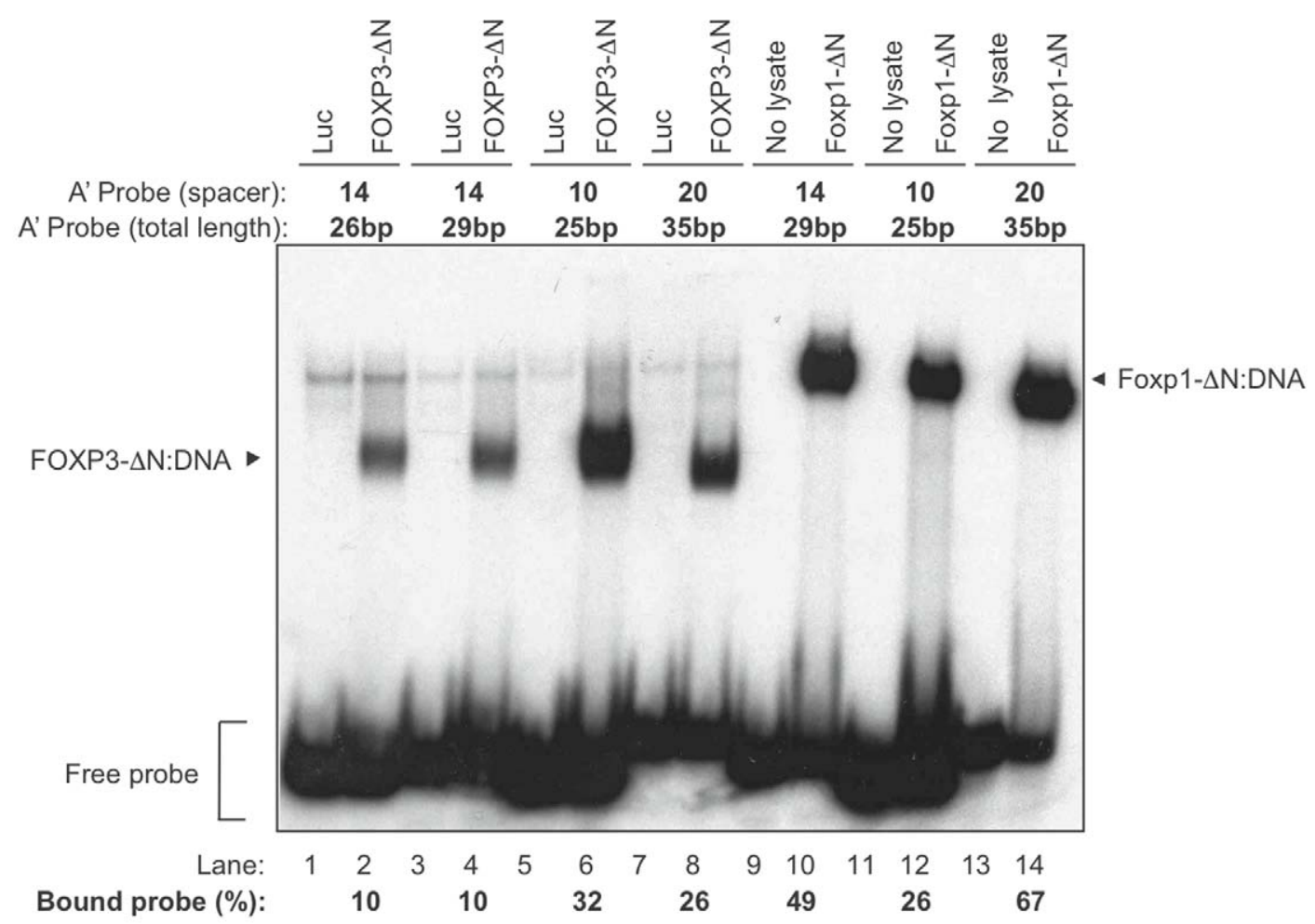

Figure 4. FOXP3:DNA binding is regulated by spacing between tandem elements. (A) Sequences of the oligonucleotides used as probes in EMSA assays. Each FOXP3 binding site is underlined with the first two nucleotides of each site highlighted in red text. The space (in number of nucleotides) separating the $5^{\prime}$ ends of the two binding sites is indicated below each sequence. The total number of base pairs in each sequence is listed next to the probe name in parentheses. (B) In vitro-translated firefly luciferase (Luc), or FOXP3- $\Delta \mathrm{N}$ were incubated with each labeled probe as indicated. Protein:DNA complexes and free probe are indicated. Quantification values of bound probe were indicated below the lanes. All data shown are representative of at least 2 separate experiments.

doi:10.1371/journal.pone.0008109.g004 
that are presented on the same side of a DNA helix and that can be as close as $10 \mathrm{bp}$ apart. However, the structural requirements for binding differ from those of Foxpl, which seems to prefer binding sites spaced apart by more than a single helical turn.

The fact that full-length FOXP3 did not bind even to the optimized $\mathrm{A}^{\prime}$ (A1-A1) probe suggested that the N-terminal region of FOXP3 has an autoinhibitory function that restricts DNA binding in vitro. To define the boundaries of this putative region, we in vitro-translated a series of FOXP3 proteins that all retained the zinc-finger, leucine-zipper and FKH domains, but in which the Nterminus was truncated to varying extents (Figure 5A, 5B-bottom). No binding could be detected even to the spacing-optimized $\mathrm{A}^{\prime}$ (A1-A1) probe until the N-terminal 121 amino acids of FOXP3 were deleted. Truncation of the entire proline-rich $\mathrm{N}$-terminus region until amino acid 181, giving rise to FOXP3- $\Delta \mathrm{N}$, was required for strongest binding to the probe (Figure 5B, lanes 6, 7).

In addition to the DNA-binding FKH domain, FOXP3- $\Delta \mathrm{N}$ contains a zinc-finger of unknown function and a leucine-zipper domain reportedly involved in homo-multimerization (Figure $5 \mathrm{C}$ ) $[9,16,35]$. As expected, deletion of the FKH domain eliminated

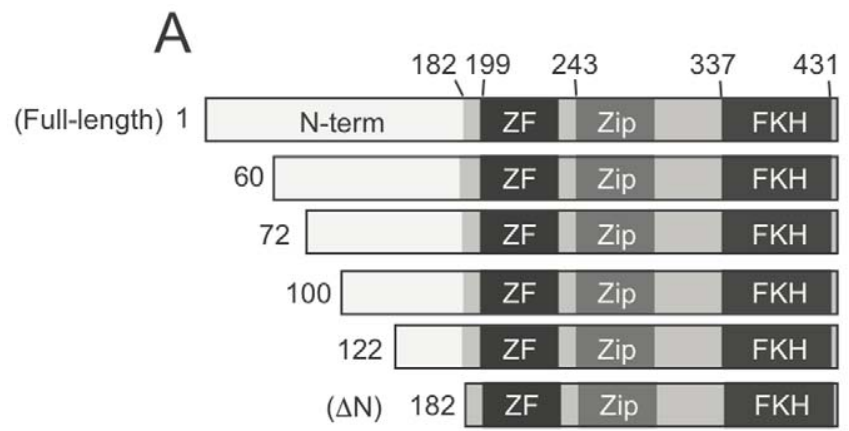

DNA-binding by FOXP3- $\Delta \mathrm{N}$ (Figure 5D, lane 1). Additionally, FOXP3- $\Delta N$ :DNA binding was abrogated by two IPEX mutations that affect the leucine-zipper domain (del K250 and del E251) [16,35,36] (Figure 5D, lanes 2, 3). These single amino acid deletions have been shown to abrogate FOXP3 multimerization [35], presumably by affecting the positioning of leucine residues along the face of the $\alpha$-helical leucine-zipper domain. In contrast, DNA binding of FOXP3- $\Delta \mathrm{N}$ was not influenced by substitution of a zinc-coordinating residue within the zinc-finger domain (C204S) (Figure 5D, lane 4); nor was binding of FOXP3- $\Delta \mathrm{N}$ enhanced by including $\mathrm{ZnSO}_{4}$ in the binding reaction (data not shown). These data indicate that the FKH domain and an intact leucine-zipper are both required for optimal DNA-binding by FOXP3- $\Delta \mathrm{N}$, whereas the zinc-finger domain is dispensable.

\section{Discussion}

FOXP3 prevents spontaneous autoimmunity by conferring on Treg cells the transcriptional profile responsible for their immunesuppressive activity. In this study we used a systematic series of

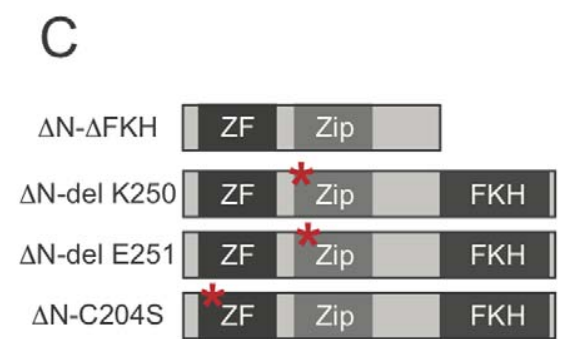

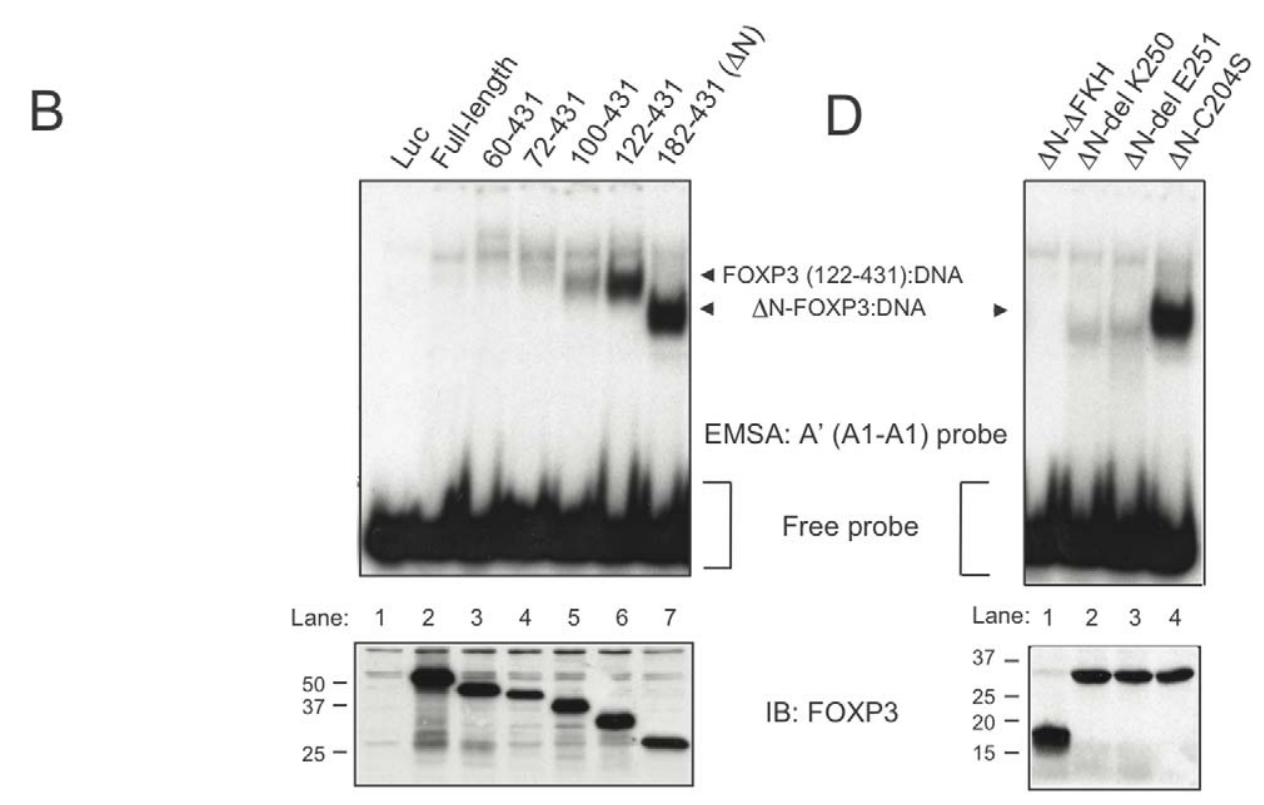

Figure 5. DNA binding by N-terminally truncated or point-mutated FOXP3. (A) Schematic representation of N-terminally truncated FOXP3 proteins used in EMSA assays. The position of the starting residue is listed next to each construct. (B) Top - Firefly luciferase (Luc), full-length FOXP3, or FOXP3 N-terminal truncation mutants were in vitro-translated and incubated with labeled $\mathrm{A}^{\prime}$ (A1-A1) probe. Arrowheads indicate Protein:DNA complexes. Bottom - expression of full-length FOXP3 or N-terminal FOXP3 mutants was determined by western blotting as in the legend to Figure 1. (C) Diagram of the FOXP3- $\Delta N$ fragments used to determine the requirement of each domain for DNA binding. A single amino acid substitution within the zinc-finger domain (C204S), or single amino acid deletions within the leucine-zipper domain (del K250, del E251) are indicated by red asterisks. (D) Top - In vitro-translated firefly luciferase (Luc), or FOXP3- $\Delta$ N mutants were incubated with labeled A' (A1-A1) probe. Arrowheads indicate Protein:DNA complexes and free probe. Bottom - expression of FOXP3- $\Delta \mathrm{N}$ mutant proteins was determined by western blotting. The same results were obtained using the $A^{\prime}(A 1-A 1)$ probe with 10-bp spacing described in Fig. 4. These data represent at least 2 independent experiments. doi:10.1371/journal.pone.0008109.g005 
EMSA assays to define the parameters that regulate high-affinity interactions between FOXP3 and cognate DNA elements. We show that the core FOXP3 consensus element contains the sequence $5^{\prime}-(\mathrm{G} / \mathrm{a})$ TAAACA $-3^{\prime}$; this sequence is also preferred by the closely related transcription factor Foxpl, but diverges from the classical FKH/FOX consensus site (5'-ATAACT-3') defined for the HNF3/FOXA proteins [32-34]. The consensus Foxp1/ FOXP3 binding element we have defined here is substantiated by previous studies that identified Foxp3 binding sites throughout the genome via ChIP-chip [29,30]. In these reports, the predicted Foxp3 binding sequence was $5^{\prime}-(\mathrm{A} / \mathrm{G})(\mathrm{T} / \mathrm{C}) \mathrm{AAACA}-3^{\prime}$. Our analysis has further defined the sequence specificity of FOXP3 as strongly preferring thymine at position 2 and favoring guanine at position 1 , although adenine is also tolerated.

The degenerate nature of FOXP3 binding sites in vivo $[29,30]$ may reflect the contributions of additional co-factors at specific loci. This hypothesis is supported by previous work showing that FOXP2 and FOXP3 can bind DNA at a non-consensus site in the Il2 promoter (5'-TGTTTCA-3') [25]. The complement of this sequence, 5'-TGAAACA-3', matches the FOXP3 binding sequence defined here except for inversion of the order of the first two nucleotides [25], which on its own would be predicted to be non-permissive for FOXP3:DNA binding. However, this site is located immediately adjacent to an NFAT site in the composite ARRE-2 element that also binds NFAT:AP-1 complexes, and the strong cooperative complexes formed between NFAT:AP-1 and NFAT:FOXP3 at this composite element promote and inhibit $I l 2$ gene transcription respectively [25]. Thus, although FOXP3 binding in vitro is restricted to its defined consensus site and requires tandem binding elements, cooperative DNA binding by FOXP3 in complex with other transcription factors may stabilize FOXP3 binding at non-consensus sites [25,26,28].

Unlike the HNF3/FOXA proteins, which bind with high affinity as monomers to single consensus sites [33], we show here that Foxpl and FOXP3 preferentially bind oligonucleotides containing two consensus sites arrayed in tandem. These results suggest that FOXP proteins bind DNA as at least a dimer [34]. In further support of this hypothesis, we find that DNA binding by FOXP3 requires the FKH domain together with an intact leucinezipper domain, whereas the zinc-finger domain is dispensable. The leucine-zipper domain of FOXP3 mediates homotypic interactions [35], whereas HNF3/FOXA proteins lack a leucine-zipper $[4,9,33]$. Therefore, the presence of a leucine-zipper domain within FOX proteins correlates with their distinctive preference for tandem sequences in DNA. Indeed, at least two independent single amino acid deletions within the leucine-zipper of FOXP3 (del K250, del E251), both associated with IPEX [16,36], fail to bind our optimized DNA sequence in EMSA assays (Figure 5D). Our data therefore suggest that the primary loss of function in these IPEX mutants relates to dimerization and DNA binding.

\section{References}

1. Kaestner KH, Knochel W, Martinez DE (2000) Unified nomenclature for the winged helix/forkhead transcription factors. Genes Dev 14(2): 142-146.

2. Friedman JR, Kaestner KH (2006) The Foxa family of transcription factors in development and metabolism. Cell Mol Life Sci 63(19-20): 2317-2328.

3. Lee CS, Friedman JR, Fulmer JT, Kaestner KH (2005) The initiation of liver development is dependent on Foxa transcription factors. Nature 435(7044): 944-947.

4. Lehmann OJ, Sowden JC, Carlsson P, Jordan T, Bhattacharya SS (2003) Fox's in development and disease. Trends Genet 19(6): 339-344.

5. Nehls M, Pfeifer D, Schorpp M, Hedrich H, Boehm T (1994) New member of the winged-helix protein family disrupted in mouse and rat nude mutations. Nature 372(6501): 103-107.

6. Gross DN, van den Heuvel AP, Birnbaum MJ (2008) The role of FoxO in the regulation of metabolism. Oncogene 27(16): 2320-2336.
Interestingly, FOXP3:DNA binding in vitro was only detected upon removal of the N-terminal region $(\mathrm{FOXP} 3-\Delta \mathrm{N})$; truncating the corresponding $\mathrm{N}$-terminal region of Foxpl also enhanced DNA binding. A trivial possibility is that the N-terminal region is unstructured and interferes, in the in vitro-translated protein, with DNA binding or multimerization. Another, more interesting possibility is that the N-terminal region of FOXP3 possesses an autoinhibitory function, possibly regulating FOXP3:DNA binding indirectly. Sequence comparisons between the N-terminal regions of FOXP proteins indicate significant divergence (reviewed in [1]). For example, the Foxpl N-terminal region contains a polyglutamine (poly-Q) sequence that is absent from the N-terminus of FOXP3 [34]. Furthermore, this N-terminal region of FOXP3 is responsible for activation as well as repression of target genes $[25,35]$, and has been shown to interact with a number of auxiliary transcription factors and chromatin-modifying proteins [39]. Thus, N-terminal sequence divergence among FOXP proteins may serve to recruit unique protein complexes to target promoters, which in turn would dictate whether gene transcription is activated or repressed. Consistent with this notion, previous reports have shown that FOXP3:DNA binding is increased, in a cyclosporine A-sensitive manner, upon stimulation of $\mathrm{T}$ cells through the $\mathrm{T}$ cell antigen receptor $[25,30,40]$. A plausible hypothesis is that the $\mathrm{N}$ terminal region of FOXP3 regulates DNA binding and transcriptional activity, either through co-factors that bind this region or through post-translational modification.

In summary, we have defined an optimal set of in vitro conditions to study FOXP3:DNA binding: (1) by removing the proline-rich $\mathrm{N}$-terminal region of FOXP3, and (2) by using an optimized probe containing two consensus sites, 5'- GTAAACA-3' separated by one or two turns of the DNA helix. Our findings will facilitate further structural studies of FOXP3 in complex with DNA, promoting a precise biochemical understanding of how FOXP3 binds to DNA, either alone or in cooperation with its transcriptional partners, to regulate the expression of target genes in Treg cells.

\section{Acknowledgments}

We thank Dr. Steven Zeigler for generously providing FOXP3 antisera and Dr. Phillip Tucker for HA-tagged murine FoxplA cDNA. We also acknowledge members of the Rao lab and Dr. Lin Chen for valuable discussion.

\section{Author Contributions}

Conceived and designed the experiments: KPK MSS AR. Performed the experiments: KPK MSS. Analyzed the data: KPK MSS AR. Contributed reagents/materials/analysis tools: KPK MSS AR. Wrote the paper: KPK MSS AR. Received funding for the work: AR.

7. Fisher SE, Scharff G (2009) FOXP2 as a molecular window into speech and language. Trends Genet 25(4): 166-177.

8. Yang T, Vidarsson H, Rodrigo-Blomqvist S, Rosengren SS, Enerback S, et al. (2007) Transcriptional control of SLC26A4 is involved in Pendred syndrome and nonsyndromic enlargement of vestibular aqueduct (DFNB4). Am J Hum Genet 80(6): 1055-1063.

9. Coffer PJ, Burgering BM (2004) Forkhead-box transcription factors and their role in the immune system. Nat Rev Immunol 4(11): 889-899.

10. Dengler HS, Baracho GV, Omori SA, Bruckner S, Arden KC, et al. (2008) Distinct functions for the transcription factor Foxol at various stages of $\mathrm{B}$ cell differentiation. Nat Immunol 9(12): 1388-1398.

11. Amin RH, Schlissel MS (2008) Foxol directly regulates the transcription of recombination-activating genes during B cell development. Nat Immunol 9(6): 613-622. 
12. Hinman RM, Nichols WA, Diaz TM, Gallardo TD, Castrillon DH, et al. (2009) Foxo3-/ - mice demonstrate reduced numbers of pre-B and recirculating $\mathrm{B}$ cells but normal splenic B cell sub-population distribution. Int Immunol 21(7): 831-842.

13. Hu H, Wang B, Borde M, Nardone J, Maika S, et al. (2006) Foxpl is an essential transcriptional regulator of B cell development. Nat Immunol 7(8): 819-826.

14. Lin L, Spoor MS, Gerth AJ, Brody SL, Peng SL (2004a) Modulation of Th1 activation and inflammation by the NF-kappaB repressor Foxj1. Science 303(5660): 1017-1020.

15. Lin L, Hron JD, Peng SL (2004b) Regulation of NF-kappaB, Th activation, and autoinflammation by the forkhead transcription factor Foxo3a. Immunity 21(2): 203-213.

16. Ziegler SF (2006) FOXP3: of mice and men. Annu Rev Immunol 24: 209-226.

17. Fontenot JD, Rudensky AY (2005) A well adapted regulatory contrivance: regulatory $\mathrm{T}$ cell development and the forkhead family transcription factor Foxp3. Nat Immunol 6(4): 331-337.

18. Brunkow ME, Jeffery EW, Hjerrild KA, Paeper B, Clark LB, et al. (2001) Disruption of a new forkhead/winged-helix protein, scurfin, results in the fatal lymphoproliferative disorder of the scurfy mouse. Nat Genet 27(1): 68-73.

19. Bennett CL, Christie J, Ramsdell F, Brunkow ME, Ferguson PJ, et al. (2001) The immune dysregulation, polyendocrinopathy, enteropathy, X-linked syndrome (IPEX) is caused by mutations of FOXP3. Nat Genet 27(1): 20-21.

20. Gambineri E, Torgerson TR, Ochs HD (2003) Immune dysregulation, polyendocrinopathy, enteropathy, and X-linked inheritance (IPEX), a syndrome of systemic autoimmunity caused by mutations of FOXP3, a critical regulator of T-cell homeostasis. Curr Opin Rheumatol 15(4): 430-435.

21. Khattri R, Cox T, Yasayko SA, Ramsdell F (2003) An essential role for Scurfin in CD4+CD25+ T regulatory cells. Nat Immunol 4(4): 337-342.

22. Godfrey VL, Wilkinson JE, Russell LB (1991) X-linked lymphoreticular disease in the scurfy (sf) mutant mouse. Am J Pathol 138(6): 1379-1387.

23. Fontenot JD, Gavin MA, Rudensky AY (2003) Foxp3 programs the development and function of CD4+CD25+ regulatory T cells. Nat Immunol 4(4): 330-336.

24. Kim JM, Rasmussen JP, Rudensky AY (2007) Regulatory T cells prevent catastrophic autoimmunity throughout the lifespan of mice. Nat Immunol 8(2): 191-197.

25. Wu Y, Borde M, Heissmeyer V, Feuerer M, Lapan AD, et al. (2006) FOXP3 controls regulatory $\mathrm{T}$ cell function through cooperation with NFAT. Cell 126(2): 375-387.

26. Ono M, Yaguchi H, Ohkura N, Kitabayashi I, Nagamura Y, et al. (2007) Foxp3 controls regulatory T-cell function by interacting with AML1/Runx1. Nature 446(7136): 685-689.
27. Zheng Y, Chaudhry A, Kas A, deRoos P, Kim JM, et al. (2009) Regulatory T-cell suppressor program co-opts transcription factor IRF4 to control $\mathrm{T}(\mathrm{H}) 2$ responses. Nature 458(7236): 351-356.

28. Bettelli E, Dastrange M, Oukka M (2005) Foxp3 interacts with nuclear factor of activated $\mathrm{T}$ cells and NF-kappa $\mathrm{B}$ to repress cytokine gene expression and effector functions of $\mathrm{T}$ helper cells. Proc Natl Acad Sci U S A 102(14): 5138-5143.

29. Zheng Y, Josefowicz SZ, Kas A, Chu TT, Gavin MA, et al. (2007) Genome-wide analysis of Foxp3 target genes in developing and mature regulatory $\mathrm{T}$ cells. Nature 445(7130): 936-940.

30. Marson A, Kretschmer K, Frampton GM, Jacobsen ES, Polansky JK, et al. (2007) Foxp3 occupancy and regulation of key target genes during T-cell stimulation. Nature 445(7130): 931-935.

31. Nardone J, Lee DU, Ansel KM, Rao A (2004) Bioinformatics for the 'bench biologist': how to find regulatory regions in genomic DNA. Nat Immunol 5(8): 768-774.

32. Costa RH, Grayson DR, Darnell JE Jr (1989) Multiple hepatocyte-enriched nuclear factors function in the regulation of transthyretin and alpha 1-antitrypsin genes. Mol Cell Biol 9(4): 1415-1425.

33. Li C, Tucker PW (1993) DNA-binding properties and secondary structural model of the hepatocyte nuclear factor 3 /fork head domain. Proc Natl Acad Sci U S A 90(24): 11583-11587.

34. Wang B, Lin D, Li C, Tucker P (2003) Multiple domains define the expression and regulatory properties of Foxpl forkhead transcriptional repressors. J Biol Chem 278(27): 24259-24268.

35. Lopes JE, Torgerson TR, Schubert LA, Anover SD, Ocheltree EL, et al. (2006) Analysis of FOXP3 reveals multiple domains required for its function as a transcriptional repressor. J Immunol 177(5): 3133-3142.

36. Ochs HD, Ziegler SF, Torgerson TR (2005) FOXP3 acts as a rheostat of the immune response. Immunol Rev 203: 156-164.

37. Gavin MA, Rasmussen JP, Fontenot JD, Vasta V, Manganiello VC, et al. (2007) Foxp3-dependent programme of regulatory T-cell differentiation. Nature 445(7129): 771-775.

38. Hori S, Nomura T, Sakaguchi S (2003) Control of regulatory T cell development by the transcription factor Foxp3. Science 299(5609): 1057-1061.

39. Zhou Z, Song X, Li B, Greene MI (2008) FOXP3 and its partners: structural and biochemical insights into the regulation of FOXP3 activity. Immunol Res 42(1-3): 19-28.

40. Chen C, Rowell EA, Thomas RM, Hancock WW, Wells AD (2006) Transcriptional regulation by Foxp3 is associated with direct promoter occupancy and modulation of histone acetylation. J Biol Chem 281(48): 36828-36834 